\title{
Know Pain Know Gain: proposing a treatment approach for phantom limb pain
}

\author{
Peter Le Feuvre, ${ }^{1}$ D Aldington ${ }^{2}$
}

${ }^{1}$ Centre for Complex Trauma DMRC Headley Court, Epsom, Surrey, UK

${ }^{2}$ DMRC Headley Court, Epsom, Surrey, UK

\section{Correspondence to} Major Peter Le Feuvre, Centre for Complex Trauma, DMRC Headley Court, Epsom, Surrey KT18 6JW, UK: 723lefeu@armymail.mod.uk

Received 5 July 2013 Accepted 8 July 2013 Published Online First 31 July 2013

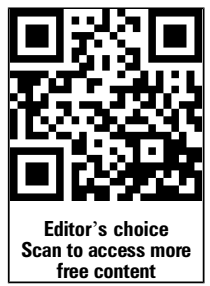

To cite: Le Feuvre $P$, Aldington D. J R Army Med Corps 2014;160:16-21.

\section{ABSTRACT}

Phantom limb pain affects between 50 and $80 \%$ of amputees. With an increasing number of battle casualties having had an amputation after combat trauma, it is inevitable that both primary and secondary care clinicians will come into contact with a patient with phantom limb pain (PLP). It is widely acknowledged that its complex aetiology means that this condition is often poorly understood and difficult to manage. A growing pathophysiological understanding is shedding new light on the mechanisms which underlie PLP. Knowledge of these mechanisms will inform treatment and enable clinicians to plan and implement solutions which make a difference to those individuals with this condition. This paper seeks to outline current research into this condition and proposes an approach to treatment. This approach has been formulated from an amalgamation of clinical experience working with battle casualties at the Defence Medical Rehabilitation Centre, Headley Court.

\section{INTRODUCTION}

The poly-trauma injury pattern resulting from blast is well documented. ${ }^{1}$ Wounding to vulnerable extremities is common and if limb salvage is unattainable, amputation will result. After amputation, phantom limb pain (PLP) affects between 50 and $80 \%$ of patients and its incidence is not related to the cause of the amputation. ${ }^{2}$

The aetiology of PLP is complex and it is often mistaken as residual limb pain (RLP) or phantom sensation. This, together with a lack of a definitive treatment, results in under-reporting of pain by patients and a lack of attention to the problem by clinicians. $^{3}$ The potential for this condition to become chronic is well documented and it can have catastrophic effects upon an individual. It is essential that both primary and secondary care clinicians understand and attend to it appropriately.

A growing pathophysiological understanding of PLP is shedding new light on the mechanisms involved. The purpose of this paper is to explore the interplay of factors involved in PLP and propose a multidisciplinary approach to treatment for first-line management within primary and secondary care. The treatment approach has been formulated from the clinical experience of working with battle casualties at the Defence Medical Rehabilitation Centre (DMRC), Headley Court and the concurrent review of literature which has informed practice. Thus, this work is applicable to those who have had a traumatic amputation rather than an amputation resulting from a chronic health condition. Treatment options are also proposed to furnish the reader with ideas which they can investigate further.
Key messages

Changes within the central nervous system contribute to the experience of phantom limb pain (PLP). Peripheral and psychological influences also act to mediate symptoms.

- The assessment should seek to highlight whether central, peripheral or psychological factors are the predominant driver in this condition.

- The treatment plan should be individualised and driven by a multidisciplinary treatment approach.

- Continuance of PLP is associated with changes in the somatosensory cortex. Stimulation of the amputation zone within the cortex appears to reverse these changes.

- Reversal of cortical changes is a key consideration within the treatment plan.

\section{CHARACTERISTICS OF PHANTOM LIMB PAIN}

PLP is often confused with pain or sensation in the areas adjacent to the amputated body part. This is known as residual limb (RLP) or stump pain and its intensity is often positively correlated with PLP. $^{4}$ Post-amputation pain at the wound site should also be distinguished from pain in the residual limb and the phantom limb. After amputation, all three may occur together. ${ }^{4}$

PLP is classified as neuropathic pain, whereas RLP and post-amputation pain are classified as nociceptive pain. PLP is often more intense in the distal portion of the phantom limb and can be exacerbated or elicited by physical factors (pressure on the residual limb, time of day, weather) and psychological factors, such as emotional stress. ${ }^{5}$ Commonly used descriptors include sharp, cramping, burning, electric, jumping, crushing and cramping. ${ }^{3}$ Often the pain is associated with particular movements or positions of the phantom limb. It differs from phantom limb sensation, which is a normal phenomenon for almost all amputees, but unlike PLP, is not distressing. ${ }^{6}$ Phantom sensation has been described as itchiness, tingling or pins and needles, squeezing and toe crossing. ${ }^{3}$

Some patients with PLP experience 'telescoping', which is simply the retraction or disappearance of the phantom limb into the residual limb. ${ }^{4}$ Traditionally, this has been seen as a positive feature. However, some evidence suggests that telescoping is a feature of central adaptations which contributes to, rather than lessens, PLP. 


\section{AETIOLOGY OF PHANTOM LIMB PAIN}

The pathophysiological understanding of PLP is growing. Neuroanatomical and neurophysiological models have shown that the brain is capable of plastic changes within the primary somatosensory cortex in response to neuropathic and musculoskeletal pain. ${ }^{8}$ Peripheral and central changes have been seen in those with chronic PLP and these changes appear to be related to the reorganisation of the cortical map. ${ }^{9}$ This reorganisation, in turn, affects perceptual, motor and autonomic systems. ${ }^{9}$ Studies using functional MRI (fMRI) suggest that those who report the greatest incidence of PLP appear to present with the greatest cortical reorganisation, while treatments which reverse this process provide relief. ${ }^{2}$ Meanwhile, psychological factors, although not the cause, seem to affect the course and severity of the pain. In short, changes within the central nervous system contribute to the experience of PLP; however, peripheral and psychological influences will also mediate symptoms. A greater understanding of these factors will inform the direction of treatment. $^{4}$

\section{Central factors}

Central sensitisation occurs when increased activity from peripheral nociceptors leads to a permanent change in the synaptic structure of the dorsal horn. Dorsal horn neurons increase in excitability, while the inhibitory process is dampened. The central nerve endings of the primary sensory neurons, interneurons and projection neurons also structurally alter. ${ }^{4} 10$

Evidence from fMRI studies has also shown that after amputation, functional and structural changes occur within the architecture of the primary somatosensory cortex. A shift of the cortical representation from neighbouring areas into the deafferented cortical amputation zone can occur. ${ }^{8}$ For example, in upper limb amputees, the greater the shift of the mouth and face representation into the deafferented hand and arm amputation zone, the greater the PLP. ${ }^{8}$ Stimulation of facial muscles, including mastication or eye movements, will then elicit PLP. In lower limb amputations this phenomenon can manifest in the migration of the representation areas for the bladder, bowel and genitals into the amputation zone. Again, stimulation of these organs will elicit PLP. Conversely, restoring normal activation within the amputation zone will reduce PLP as it appears to reduce the migration of neighbouring areas into the cortical amputation zone. ${ }^{5}$ Restoration of activity within these areas of the somatosensory cortex appears to involve visual, sensory and motor stimuli. Myoelectric prosthesis, sensory discrimination, motor imagery and mirror therapy are some of the methods which have shown success. $^{2} 811$

\section{Peripheral factors}

Nociceptive input from the residual limb appears to correlate with the level of PLP. ${ }^{4}$ Regenerative sprouting of the axon occurs when a peripheral nerve is cut, and a neuroma in the residual limb may form. Ectopic discharge from a neuroma is one nociceptive signal which may in turn stimulate a neuropathic response in the form of PLP. Other causes can include wound infection, osteomyelitis and poor prosthetic fit. In our military patient group the presence of heterogenic ossification (HO) is well documented. ${ }^{12}$ The development of $\mathrm{HO}$ can be an additional nociceptive aggravator.

PLP is, however, present in patients immediately after amputation before a neuroma or HO can form and even local anaesthesia may not eliminate it. ${ }^{4}$ Anaesthetic block of a neuroma eliminates discharge from the residual limb; however, it will not affect ectopic discharge emanating from the dorsal root ganglion. ${ }^{10}$ The dorsal root ganglion can amplify discharge from the residual limb or cross-excite neighbouring neurons. Increased circulating epinephrine resulting from sympathetic discharge will also trigger or exacerbate neuronal activity. Such sympathetic discharge can result from emotional distress, and may also be due to temperature or inflammation. ${ }^{4}$ When sympathetic activity is blocked, PLP may reduce, whereas an injection of epinephrine will increase PLP. ${ }^{4}$ In short, sympathetic discharge may act as a 'volume control' to what is already happening to the patient.

\section{Psychological factors}

Patients with PLP present with a normal psychological profile. ${ }^{13}$ Pre-existing psychological variables before amputation, deficient coping strategies and stress have all been reported to evoke and modulate PLP. The sympathetic nervous system or muscular response to stress may well be the primary mediator. ${ }^{4}$

\section{Interaction of factors}

PLP exists even when there is no pathology in the residual limb. Birbaumer et al used an anaesthetic block for upper limb amputees with PLP; 50\% found relief and reversal of cortical reorganisation was demonstrated. ${ }^{14}$ In these patients, peripheral factors were important in the maintenance of PLP. It was suggested that in the remaining 50\% the process of cortical reorganisation had become independent of peripheral factors. ${ }^{14}$ Hence, peripheral factors whether regional abnormalities or a sympathetic dysregulation are not the whole picture, but nor are central factors; both appear to have a role.

However, Birbaumer's findings may also suggest that progressive sensitisation exists, so at the point of amputation, peripheral factors dominate, but if this continues, central factors become the predominant influence. Flor argues that the implicit changes within the primary somatosensory cortex are in response to enduring nociceptive input or the loss of input altogether, ${ }^{4}$ so a situation involving central adaptation and peripheral sensitisation in response to amputation is possible. The implication of this argument is that no single treatment is likely to be the answer. Instead, a treatment philosophy is required, which acknowledges the varying interplay of central, peripheral and psychological factors. How such a philosophy may look in practice will be considered.

\section{TREATMENT APPROACH}

Most studies investigating the effectiveness of treatment for PLP have been short-term, uncontrolled trials using a small or disparate sample population. Even larger studies and surveys of patients have not accounted for the mechanism underlying the production of pain, and so robust data are lacking. ${ }^{4}$ The lack of comparative trials and the complex individual multifactorial drivers therefore demand a multidisciplinary approach. The treatment approach (Figure 1) and treatment table (Figure 2) emphasise a multidisciplinary solution when treating this condition.

\section{Assessment}

The assessment must accurately diagnose the source(s) of the pain. A failure to discriminate between RLP, PLP and phantom limb sensation means that the patient will not receive the appropriate care.

It is accepted that clinically significant cortical reorganisation is associated with continuing chronic PLP. These central changes are due to enduring nociceptive input, loss of input to the 


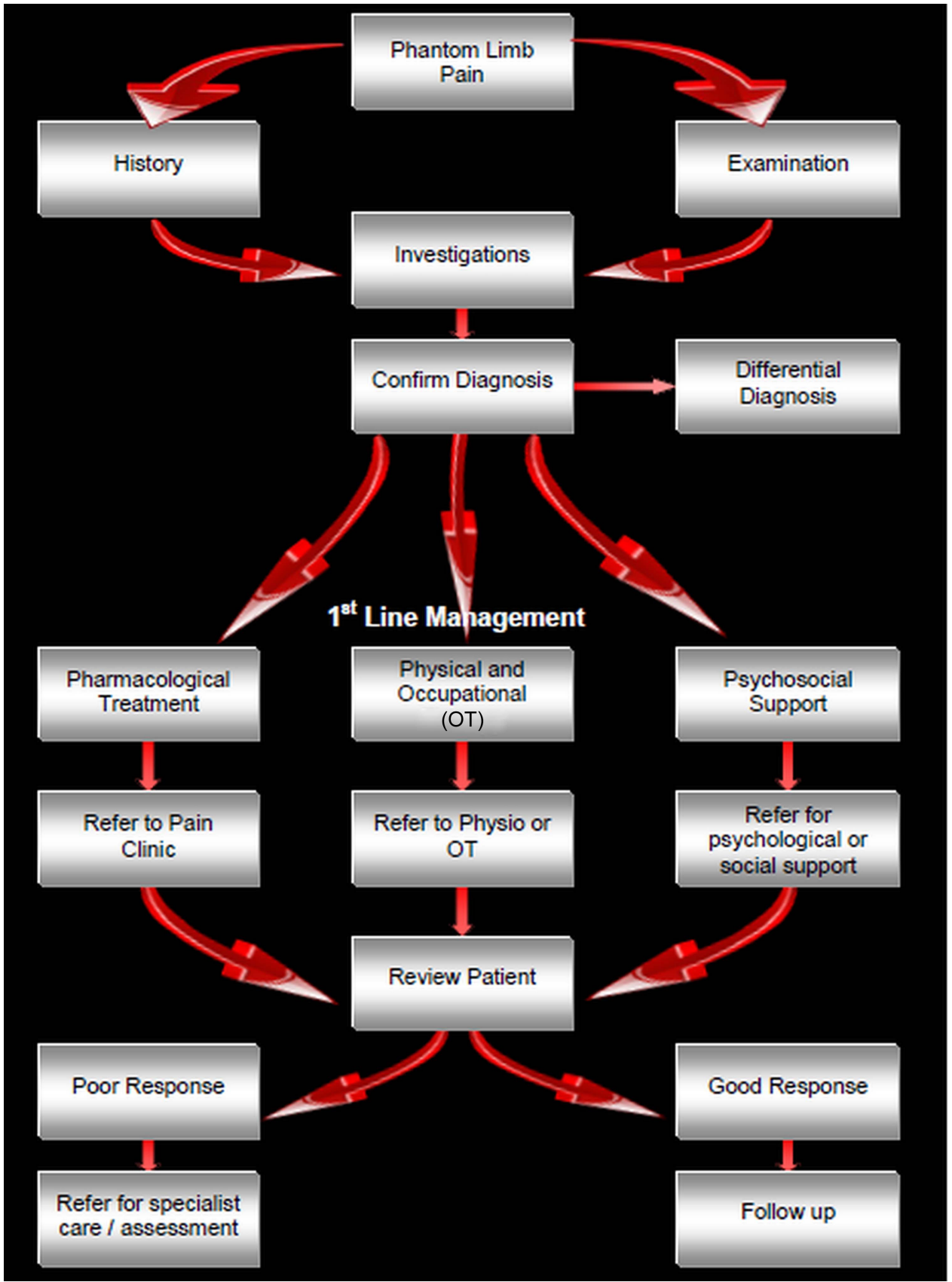

Figure 1 A pathway for management of phantom limb pain.

cortical zone of amputation or a combination of both. Therefore, to prevent chronicity, effective treatment must eliminate the nociceptive stimuli and restore normal input to the cortical zone of amputation. The assessment must enlighten the clinician about the degree of interplay between these two factors. It should also explore sources of enduring nociceptive input and sympathetic discharge from psychosocial factors, as well as documenting cortical changes which might have already occurred. The assessment should highlight which factor(s) is the dominant driver of the person's PLP: central, peripheral or psychological. In this way, investigation and treatment can focus on the dominant factor.

\section{Differential diagnosis}

Simply discriminating between RLP and PLP is more complex than it appears. Both often coexist and RLP may provoke PLP.
Eliminating the causes of RLP is therefore the priority as this will resolve or lessen PLP which is respondent to peripheral aggravators. It also shows the degree to which central factors may have an ongoing influence.

Immediate post-amputation management demands early effective analgesia and adjunctive measures, include managing oedema using elastic stump socks, semi-rigid dressings and rigid plaster casts. ${ }^{12}$ Post-acute management requires attention to both intrinsic and extrinsic causes of RLP.

Extrinsic RLP will result from complications of wound healing and so infection must be excluded. Tissue load and sheering forces placed on the limb due to a poor prosthetic fit will also evoke pain. A prosthetic review will improve fit and enable sensitised structures to be offloaded. Scar formation can also cause pain, particularly where there is nerve entrapment, or adhesions reducing the mobility of soft tissues. In either case, 


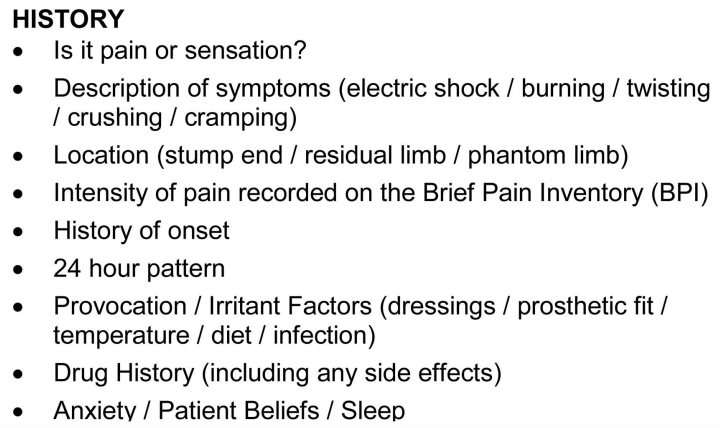

- Description of symptoms (electric shock / burning / twisting / crushing / cramping)

- Location (stump end / residual limb / phantom limb)

- Intensity of pain recorded on the Brief Pain Inventory (BPI)

- History of onset

- 24 hour pattern

- Provocation / Irritant Factors (dressings / prosthetic fit / temperature / diet / infection)

- Drug History (including any side effects)

- Anxiety / Patient Beliefs / Sleep

\begin{tabular}{|c|c|c|}
\hline INVESTIGATIONS & PHANTOM LIMB PAIN PATHWAY & DIFFERENTIAL DIAGNOSIS \\
\hline X-Ray & & Residual Limb Pain \\
\hline CT / MRI Scan & & Heterogenic ossification $(\mathrm{HO})$ \\
\hline Nerve conduction testing & nation & Infection \\
\hline Bloods & & Neuroma / Neural tethering \\
\hline Arterial & & Hysteria or malingering \\
\hline Swab of wound & ferential & Deep vein thrombosis \\
\hline Ultra-sound & & Musculoskeletal injury \\
\hline $\begin{array}{l}\text { FIRST LINE } \\
\text { PHARMACOLOGICAL } \\
\text { TREATMENT: }\end{array}$ & That & $\begin{array}{l}\text { PSYCHOSOCIAL SUPPORT } \\
\text { Pre-existing or concurrent } \\
\text { psychological or psychosocial }\end{array}$ \\
\hline $\begin{array}{l}\text { Amitriptyline: } 25 \mathrm{mgs} \text { up to } \\
100 \mathrm{mgs} \text { at night*. }\end{array}$ & & $\begin{array}{l}\text { issues may present, and PLP } \\
\text { may itself precipitate }\end{array}$ \\
\hline $\begin{array}{l}\text { Pregabalin } 75 \mathrm{mgs} \text { bd up to } \\
300 \mathrm{mgs} \text { bd }\end{array}$ & & Management options: \\
\hline $\begin{array}{l}{ }^{*} \text { Higher doses and } \\
\text { alternatives are possible } \\
\text { following specialist advice }\end{array}$ & $\begin{array}{c}\text { Refer for } \\
\text { specialist care } \\
\text { nis }\end{array}$ & $\begin{array}{l}\text { therapy } \\
\text { - family / social support } \\
\text { - EMDR }\end{array}$ \\
\hline
\end{tabular}

PHYSICAL AND OCCUPATIONAL THERAPY (in no particular order)

$\begin{array}{ll}\text { 1. Irritant Management: Attend to or highlight causes: } & \text { 6. Physical Exercise } \\ \text { (dressings, infection, swelling, drug side effects, } & \text { 7. Scar Management } \\ \text { prosthetic fitting, HO, neuroma, adhesions). } & \text { 8. Graded motor imagery } \\ \text { 2. Application of compression to residual limb } & \text { (Laterality - Motor imagery - Mirror Therapy) } \\ \text { (Juzo, tubifast, prosthesis) } & \text { 9. Acupuncture } \\ \text { 3. Education and re-assurance (10) } & \text { 10 Cognitive Behavioural Therapy (CBT) } \\ \text { 4. Limb massage / De-sensitisation } & 11 \text { Heat / Ice } \\ \text { 5. TNS Machine } & \text { 12 Relax Sock } \\ & \text { 13. Trigger Point release in the residual limb }\end{array}$

Figure 2 A summary of treatment for phantom limb pain (PLP). EMDR, eye movement desensitisation and reprocessing; TNS, transcutaneous nerve stimulation.

scar management using soft tissue massage and moisturiser is recommended; silicone treatment can also be added if required. ${ }^{16}$ Besides improving tissue mobility, massage can be used to desensitise the residual limb. ${ }^{17}$

Intrinsic causes of RLP can include ischaemia, joint dysfunction proximal to the residual limb, stress fracture, osteomyelitis and wound dehiscence. Occasionally where the bone has been improperly trimmed or formation of bone in extraskeletal soft tissue has occurred (HO), then pain may result in high-pressure areas. ${ }^{12}$ Investigations will be required and revision surgery may be considered; alternatively, prosthetic adjustment can be used to unload pressure areas.

Neuroma is the most common cause of intrinsic RLP. ${ }^{12}$ Ectopic discharge may evoke a neuropathic response causing PLP. ${ }^{4}$ Neuroma formation after amputation is normal, but when it becomes sensitised to mechanical or chemical stimuli, often exacerbated by entrapment, then problems ensue. ${ }^{12}$ Pain is intermittent and variable, but diagnosis is confirmed by a specific site
EXAMINATION

- Examine skin for warmth, swelling, signs of infection.

- Examine stump end - consider $\mathrm{HO} /$ Neuroma (specific point of pain) / wound breakdown.

- Range of movement of residual $\operatorname{limb}(\mathrm{s})$.

- Power of residual limb(s).

- Examine sources of musculoskeleta referral (lower back / hip / cervical spine / shoulder)

of tenderness on palpation, which can be confirmed with an injection of local anaesthetic into the site. ${ }^{12}$ Surgical referral can be considered, but massage, vibration, acupuncture and transcutaneous electrical nerve stimulation (TENS) may also effectively desensitise the area. ${ }^{12}$

\section{Pharmacological management}

Simple non-steroidal, anti-inflammatory and opioid drugs can be tried, but the mainstay of the pharmacological management of PLP is the use of antineuropathic medication. UK military pain management system encourages the use of pregabalin and amitriptyline as early as possible; it might have been started before returning to the UK after injury.

First-line treatment is a trial of up to $300 \mathrm{mg}$ twice daily of pregabalin and up to $150 \mathrm{mg}$ of amitriptyline at night. If pregabalin is insufficient, or depression is a problem, duloxetine may be used. Opioids are of variable help, particularly with these 
pains. It may be that tapentadol will prove to be beneficial, but it is too early to say clearly.

However, while pharmacological agents can be of use, the way they are used is even more important. The patient has to understand that the agents are not going to remove all pain. Indeed the degree of pain relief from the agents is almost irrelevant. What really matters is that the agents enable the patient to 'do more'. In this way they can be likened to the old confectionary advertisement that suggested it allows you to 'work, rest and play'; the point being if the pharmacological agents do not have this action there is no point taking them.

As with many pains, there appear to be two fundamental problems in the pharmacological management of PLP. The first, is that patients often have to wait to be allowed to try the medication and getting the dose right may also take a while. Most pharmacological dose regimens are either fixed or dependent on biomarker values; pain-related medication is far more subjective. Regular discussions with the patient can combat this problem. The second problem is getting the patients to stop medication when it is not helping. Again, patient education about what the medication can be expected to achieve, together with regular reviews, is the answer. When this technique is used, it is common for patients to recognise that medication often makes their lives worse and they will willingly stop taking it at the expense of slightly more pain.

\section{Physical and occupational therapy}

Combining physical and occupational therapy with a cognitive understanding of the condition will amplify the effects of treatment. Our management should aim to equip and empower the patient, informing them about their condition and how they can take control while seeking to alter destructive or erroneous beliefs and actions. Common self-treatment strategies can include wearing an elastic stump sock to minimise volume changes in the residual limb, stump massage, mental imagery of the phantom limb and taking physical exercise.

When attending to factors which provoke PLP, joint dysfunction proximal to the residual limb and prosthetic fit have been highlighted. However, good prosthetic use is as important. Normalising the gait pattern is, in part, due to prosthetic fit and alignment. It is also dependent on good proprioception, correct motor patterning and symmetrical movement control enabling dissociation of movement between trunk and limb. In turn, the residual limb(s), trunk and spinal segments must have sufficient range and control of movement to achieve a symmetrical gait pattern. It is important to assess all planes of movement, particularly rotation of the trunk. ${ }^{18}$ Although this practice has been extrapolated from able-bodied research, the principle of gaining proximal stability and control of the trunk in order to achieve limb mobility is more important in the amputee. Shortened limbs must control a lengthened lever (prosthesis) without causing undue stress to surrounding musculoskeletal structures in order to achieve good prosthetic use.

Prosthetic use has been associated with reduced PLP. Upper limb amputees using a myoelectric prosthesis had less cortical reorganisation and PLP than those who used a cosmetic prosthesis or none at all. ${ }^{19}$ The myoelectric prosthesis creates sensory, visual and motor feedback. The cortical amputation zone receives somatosensory and motor input, which may explain the reversal of cortical reorganisation and improvements in PLP. Lower limb amputees, do not use a myoelectric prosthesis, but prosthetic use enables normal limb movement, standing posture and gait. This may normalise visual, sensory and motor input to the deafferented amputation zone within the cortex and so reverse cortical reorganisation. This might explain our anecdotal observation that good prosthetic use is associated with less PLP.

Similarly, mirror therapy is a therapeutic intervention which has been shown to affect motor and sensory processes through the relative dominance of the visual input it provides. ${ }^{15}$ The effect is created by viewing a reflection of the intact limb through a mirror placed where the amputated limb would have existed. Most of the evidence for this intervention comes from case studies and anecdotal data. Chan et al allocated 22 patients with PLP into a mirror therapy group, mental imagery group and a covered mirror group (control). ${ }^{20}$ They reported that all patients in the mirror therapy group experienced reduced PLP. This was not the case in the other two groups. The study did not control potential biases and its methodology was not described in detail, so weakening the power of its findings. A more robust trial compared a mirror group with a covered mirror group; however, there were no statistically significant reductions in PLP between the groups. ${ }^{21}$ An example of the exercises used with the mirror box is shown in Box 1.

MacIver et al used fMRI before and after a 6-week intensive course of motor imagery with 13 upper limb amputees all of whom had PLP. ${ }^{8}$ In contrast to Chan et al, this author found a significant reduction in PLP with motor imagery and this was associated with a reversal in cortical reorganisation. ${ }^{8}$ However, again biases were poorly controlled and the sample size was small. Moseley argued that while mirrored movements may expose the cortex to sensory and motor input, the therapeutic effect is magnified if cortical networks are gradually activated using limb recognition, motor imagery and finally, mirrored movement. $^{23}$ This sequence of cortical exposure has become known as graded motor imagery. A single blind randomised control design has been used to investigate this approach for conditions such as PLP, chronic regional pain syndrome type 1 and brachial plexus avulsion. ${ }^{9} 2223$ Although the heterogeneity of the sample is acknowledged, it is argued that cortical similarities exist between these conditions, causing a cortical neglect of the affected limb leading to changes in cortical mapping. The sample size in each study was small, but all three studies showed

\section{Box 1 Unilateral lower limb mirror box exercises}

Mirrored movement exercises (10 repetitions of each) ${ }^{21}$ :

1. Slowly straighten the knee then bend both knees together.

2. Slowly straighten your legs and then bend each knee alternatively as if walking.

3. Move your feet together, point your feet upwards and then point your feet downwards.

4. Turn your soles in towards each other and then away from each other.

5. Move your feet around in a circle to the left and to the right.

6. Lift your feet off the ground in a walking movement.

7. Point your toes upwards and then downwards while keeping your ankle and foot still.

8. Clench and unclench your toes.

9. Spread your toes and then relax them.

10. Point up your big toes and point down the other toes, then reverse it so that your big toe is pointing down and your other toes are pointing up. 
significant reductions in pain and cortical reorganisation after a 6-week programme. Clinicians wishing to add this programme to their treatment repertoire can find resources at http://www. noigroup.com. As a guide, the regimen used in these trials took place over 6 weeks and the time was equally divided between the three treatments.

Black et al cite a number of placebo-controlled trials and epidemiological surveys which found that TENS may be effective at reducing PLP. ${ }^{17}$ Scar massage and percussion were also discussed as an effective way of desensitising the residual limb. These approaches may benefit patients for whom a peripheral component is the dominant feature; however, there is little evidence in the literature to aid patient selection.

Ketz surveyed US military personnel with single or multiple amputations resulting from combat operations. ${ }^{3}$ Moderate relief was found using physical exercise, relaxation and distraction, but no participants reported using alternative treatments. A number of studies have cited the potential of acupuncture to relieve $\mathrm{PLP}^{24}$ and unpublished case reports using acupuncture at DMRC certainly support this proposition. There is also good evidence that acupuncture provides somatosensory stimulation and this might be one mechanism by which it can modulate pain. ${ }^{25}$ However, again no consensus exists within the literature pertaining to its application and patient selection.

\section{Psychological support}

Patients with PLP do not have a greater prevalence of personality or psychological disorders compared with the general population. The mechanism of their injuries and premorbid psychosocial issues will influence their presentation, and sympathetic discharge will provoke PLP through its modulation of neuronal activity. Emotional reactions will stimulate sympathetic discharge, and individual cognitive evaluation of the perceived threat posed by PLP will also feed into this. In common with other chronic pain conditions, the sensory discriminative dimension (localisation) of pain will reduce over time but the cognitive affective (suffering) dimension of pain will increase. Psychosocial support is the responsibility of the whole clinical team and this aspect of care should be considered together with the medical and rehabilitative elements.

If it is assumed that the team seeks to equip and empower the patient, while altering destructive or erroneous belief patterns, then most patients should find their needs met by the multidisciplinary approach proposed. However, formal mental health referral will be required for some patients. For such patients counselling, cognitive behavioural therapy or eye movement desensitisation and reprocessing may be effective. ${ }^{26}$

\section{CONCLUSION}

The treatment of PLP demands a multidisciplinary approach which acknowledges the interplay between central, peripheral and psychological factors against a background of individual experiences and concern. The goal is to prevent cortical reorganisation in response to amputation and so prevent chronicity of this condition. The aim is to normalise visual, sensory and motor input to the central nervous system, which may be devoid of input or in receipt of altered messages. To achieve this aim, causes of ongoing nociceptive input must be tackled, antineuropathic medication must be employed at a dosage which is beneficial to the patient and cortical stimulation must be encouraged. Stimulation of the cortex may occur with good prosthetic use, acupuncture, graded motor imagery or its individual components, limb recognition, motor imagery and mirror therapy. In addition, we must acknowledge the power of the sympathetic nervous system to modulate pain and so we must not forget the importance of attending to the patient's psychosocial needs and concerns. For most patients seen in primary and secondary care, this will suffice and importantly, it will give the patient strategies to control their symptoms. When a consistent multidisciplinary approach to provide first-line management fails to resolve symptoms, then referral to specialist care is indicated. The nature of the specialist care will depend upon whether peripheral, central or psychological factors are deemed to be the cause of the continuing PLP.

Contributors Both authors have been involved in the conception and design of the manuscript; the drafting of the article and the intellectual content. The main author has also been involved in the final approval of the version to be published.

Competing interests None.

Provenance and peer review Not commissioned; externally peer reviewed.

\section{REFERENCES}

1 Sayer NA, Cifu DX, McNamee S, et al. Rehabilitation needs of combat-injured Service members admitted to the VA Polytrauma Rehabilitation Centers: the role of $P M \& R$ in the care of wounded soldiers. PM\&R 2009;1:23-8.

2 Diers $\mathrm{M}$, Christmann C, Koeppe C, et al. Mirrored, imagined and executed movements differentially activate sensorimotor cortex in amputees with and without phantom limb pain. Pain 2010;149:296-304.

3 Ketz AK. The experience of phantom limb pain in patients with combat-related traumatic amputations. Arch Phys Med Rehabil 2008;89:1127-32.

4 Flor $\mathrm{H}$. Phantom-limb pain: characteristics, causes and treatment. Lancet 2002:1:182-9.

5 Flor $\mathrm{H}$. Cortical reorganisation and chronic pain; implications for rehabilitation. J Rehabil Med 2003;41:66-72.

6 Kooijmana CM, Dijkstra PU, Geertzena JHB, et al. Phantom pain and phantom sensations in upper limb amputees: an epidemiological study. Pain 2000;87:33-41.

7 Grusser S, Winter C, Muhlnickel W, et al. The relationship of perceptual phenomena and cortical reorganization in upper extremity amputees. Neuroscience 2001;102:263-72.

8 Maclver K, Lloyd DM, Kelly $S$, et al. Phantom limb pain, cortical reorganization and the therapeutic effect of mental imagery. Brain 2008;131:2181-91.

9 Moseley GL. Graded motor imagery for pathologic pain: a randomized controlled trial. Neurology 2006;67:2129-34.

10 Butler D, Moseley GL. Explain pain. Noigroup Publications, 2010.

11 Flor $H$, Denke $C$, Schäfer $M$, et al. Effects of sensory discrimination training on cortical reorganization and phantom limb pain. Lancet 2001;357:1763-4.

12 Walsh N. Pain management for the lower limb amputee. AAOP 2005. http://www. oandp.org/publications/jop/2005/2005-39.asp

13 Sherman RA, Sherman CJ, Bruno GM. Psychological factors influencing chronic phantom limb pain: an analysis of the literature. Pain 1987;28:285-95.

14 Birbaumer $\mathrm{N}$, Lutzenberger $\mathrm{W}$, Montoya $\mathrm{P}$, et al. Effects of regional anesthesia on phantom limb pain are mirrored in changes in cortical reorganization. J Neurosci 1997; 17:5503-8.

15 Moseley GL, Gallace A, Spence C. Is mirror therapy all it is cracked up to be? Current evidence and future directions. Pain 2008;138:1387-10.

16 Mustoe TA, Cooter RD, Gold MH, et al. International clinical recommendations on scar management. Plast Reconstr Surg 2002;110:560-71.

17 Black LM, Persons RK, Jamieson MLS. What is the best way to manage phantom limb pain? J fam practice 2009;58:155-8.

18 Comerford MJ, Mottram SL. Functional stability re-training: principles and strategies for managing mechanical dysfunction. Manual Ther 2001;6:3-14.

19 Lotze M, Flor H, Grodd W, et al. Phantom movements and pain. An fMRI study in upper limb amputees. Brain 2001;124:2268-77.

20 Chan $B$, Witt $R$, Charrow A, et al. Mirror therapy for phantom limb pain. N Engl J Med 2007;357:2206-7.

21 Brodie E, Whyte A, Niven C. Analgesia through the looking glass? A randomized controlled trial investigating the effect of viewing a 'virtual' limb upon phantom limb pain, sensation and movement. Eur J Pain 2007:11:428-36.

22 Moseley GL. Is successful rehabilitation of complex regional pain syndrome due to sustained attention to the affected limb? A randomised clinical trial. Pain 2005;114:54-61.

23 Moseley GL. Graded motor imagery is effective for longstanding complex regional pain syndrome. Pain 2004;108:192-8.

24 Bradbrook D. Acupuncture treatment of phantom limb pain and phantom limb sensation in amputees. Acupunct Med 2004;22:93-7.

25 Dhond RP, Yeh C, Park K, et al. Acupuncture modulates resting state connectivity in default and sensorimotor brain networks. Pain 2008;136:407-18.

26 Schneider J, Hofmann A, Rost C, et al. EMDR in the treatment of chronic phantom limb pain. Pain Medicine 2008;9:76-82. 